\title{
Anestesia epidural em cutias (Dasyprocta azarae)
}

\author{
Epidural anesthesia in agoutis (Dasyprocta azarae) \\ Fabrício Singaretti de Oliveira', Leandro Luís Martins' ${ }^{2}$ Juan Carlos Duque ${ }^{3}$, \\ Ana Paula Pauloni ${ }^{4} \&$ Carlos Augusto Araújo Valadão ${ }^{5}$
}

\begin{abstract}
RESUMO
Uma das dificuldades no manejo de animais selvagens encontra-se na pouca informação existente sobre anestesia segura, principalmente em roedores, como a cutia. Diversos protocolos anestésicos foram testados sem sucesso na obtenção de analgesia cirúrgica, mesmo com o uso de pré-medicação com azaperone ( $4 \mathrm{mg} / \mathrm{kg})$ e meperidina $(4 \mathrm{mg} / \mathrm{kg})$ e altas doses de xilazina $(0,8 \mathrm{mg} / \mathrm{kg})$ e cetamina $(42 \mathrm{mg} / \mathrm{kg})$. Depois de alguns ensaios, observou-se que o uso de doses menores dos anestésicos junto com a técnica de anestesia epidural com lidocaína abolia a resposta voluntária a estímulos dolorosos somáticos. Assim, sete machos, adultos, pertencentes ao Zoológico Municipal de Catanduva, SP, foram tranqüilizados com azaperone (4 mg/kg) e meperidina ( $4 \mathrm{mg} / \mathrm{kg})$. Quinze minutos depois foram administradas cetamina $(20 \mathrm{mg} / \mathrm{kg})$ e xilazina $(0,4 \mathrm{mg} / \mathrm{kg})$, também pela via intra-muscular. Posteriormente, foi injetada lidocaína no espaço lombossacro, na dose de $5 \mathrm{mg} / \mathrm{kg}$. Foram avaliados o período de latência da associação anestésica (3,86 $\pm 1,87$ minutos); período de latência da lidocaína epidural (6,86 $\pm 2,85$ minutos); o tempo hábil de analgesia (80,86 $\pm 16,1$ minutos); e a temperatura retal, a qual diminuiu, em média, $1,76 \pm 0,53$ graus centígrados desde o começo da anestesia até o final do período hábil de analgesia. Todos os animais se recuperaram de forma satisfatória, sem apresentar sinais de excitação e não foram observadas complicações decorrentes da técnica epidural. Concluiu-se que a técnica de anestesia balanceada empregada proporcionou analgesia adequada, durante tempo que seria suficiente para a realização de vários procedimentos, com a utilização de doses reduzidas de xilazina e cetamina.
\end{abstract}

Descritores: anestesia, epidural, cutia, Dasyprocta azarae.

\section{ABSTRACT}

One of the greatest difficulties on management of wild species is the little data on anesthesia, mainly in rodents, as agoutis. Several anesthesic protocols were unsucesslly tested for surgical analgesia, even using premedication with azaperone $(4 \mathrm{mg} / \mathrm{kg})$ and meperidine $(4 \mathrm{mg} / \mathrm{kg})$ and high doses of xylazine $(0,8 \mathrm{mg} / \mathrm{kg})$ and ketamine $(42 \mathrm{mg} / \mathrm{kg})$. After some tests, the use of low doses of anesthesics together with epidural anesthesia with lidocaine eliminated the voluntary response to somatic painful stimuli. Thus, seven adult males, beolonging to the Catanduva City Zoo were tranquilized with azaperone azaperone (4 mg/kg) and meperidine $(4 \mathrm{mg} / \mathrm{kg})$. After 15 minutes, ketamine $(20 \mathrm{mg} / \mathrm{kg})$ and xylazine $(0.4 \mathrm{mg} / \mathrm{kg})$ were injected via intramuscular. Posteriorly, lidocaine $(5 \mathrm{mg} / \mathrm{kg}$ ) was injected in lumbosacral space. The time of latency of the anesthesic association $(3.86 \pm 1.87$ minutes); time of latency of epidural lidocaine ( $6.86 \pm 2.85$ minutes); and time of analgesia ( $80.86 \pm 16.1$ minutes) were analyzed, besides rectal temperature, which decreased, in average, $1.76 \pm 0.53^{\circ} \mathrm{C}$ from the beginning of the anesthesia to the end of the able time. All animals got satisafatory recovery, without presenting excitation signs and not observing complications due the epidural tecnique. It was concluded that the balanced anesthesia tecnique provided adequated analgesia during a time considered enough for several procedures, by means of low doses of xylazine and ketamine.

Key words: anesthesia, epidural, agoutis, Dasyprocta azarae. 


\section{INTRODUÇÃO}

A cutia é um dos grandes roedores latino-americanos, como as pacas e as capivaras [2]. Em relação à tranqüilização e anestesia desse grupo de animais, pesquisas vêm sendo realizadas visando-se estabelecer protocolos seguros para realização de procedimentos clínicos ou cirúrgicos. Entretanto, não há relatos sobre anestesia epidural nessas espécies, apenas anestesia dissociativa ou geral. Assim, o objetivo dessa pesquisa foi descrever a anestesia epidural lombossacra em cutias criadas em cativeiro, proporcionando bases para futuros experimentos.

\section{MATERIAIS E MÉTODOS}

Diversos protocolos anestésicos foram testados sem sucesso na obtenção de analgesia cirúrgica, mesmo com o uso de pré-medicação com azaperone ${ }^{1}$ $(4 \mathrm{mg} / \mathrm{kg})$ e meperidina ${ }^{2}(4 \mathrm{mg} / \mathrm{kg})$ e altas doses de xilazina $^{3}(0,8 \mathrm{mg} / \mathrm{kg})$ e cetamina ${ }^{4}(42 \mathrm{mg} / \mathrm{kg})$.

Depois de alguns ensaios, observou-se que o uso de doses menores dos anestésicos, junto com a técnica de anestesia epidural com lidocaína, abolia a resposta voluntária a estímulos dolorosos somáticos. Assim, sete machos, adultos, pertencentes ao Zoológico Municipal de Catanduva, SP, e pesando 2,69 $\pm 0,49 \mathrm{~kg}$, foram capturados e contidos em sacos de polipropileno para aferição dos parâmetros sobre efeito de anestesia.

Os animais foram tranquiilizados com azaperone $(4 \mathrm{mg} / \mathrm{kg})$ e meperidina $(4 \mathrm{mg} / \mathrm{kg})$. Quinze minutos depois foram administradas cetamina $(20 \mathrm{mg} / \mathrm{kg})$ e xilazina $(0,4 \mathrm{mg} / \mathrm{kg})$, todos os fármacos pela via intramuscular. Posteriormente, os animais foram colocados em decúbito esternal, com os membros pélvicos tracionados cranialmente, para administração da injeção epidural no espaço lombossacro, previamente identificado por palpação. Foi então injetada lidocaína ${ }^{5}$, na dose de $5 \mathrm{mg} / \mathrm{kg}$, durante um minuto. Foram avaliados o período de latência da associação anestésica (tempo entre a administração de xilazina/cetamina e o momento em que o animal permaneceu em decúbito lateral e permitiu a manipulação); período de latência da lidocaína epidural (tempo entre a injeção epidural e a perda do reflexo anal, do tônus muscular dos membros pélvicos e da resposta ao pinçamento do espaço interdigital e da falange proximal); o tempo hábil de analgesia (tempo entre a aplicação da lidocaína e o momento em que o animal respondeu de forma voluntária ao pinçamento do espaço interdigital e da falange proximal [5]; e a temperatura retal.

\section{RESULTADOS}

Os períodos de latência da associação anestésica

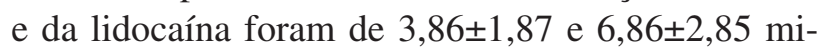
nutos, respectivamente, e o período hábil de analgesia foi de $80,86 \pm 16,1$ minutos. A temperatura retal diminuiu, em média, 1,76 $\pm 0,53$ graus centígrados desde o começo da anestesia até o final do período hábil de analgesia (Tabela 1). Todos os animais se recuperaram de forma satisfatória, sem apresentar sinais de excitação e não foram observadas complicações decorrentes da técnica epidural.

\section{DISCUSSÃO}

A escolha dos fármacos utilizados foi baseada em ampla revisão de literatura, constatando-se que os anestésicos dissociativos empregados via intramuscular foram de fácil aplicação, proporcionando boa segurança [1].

A técnica anestésica empregada nas cutias, utilizando azaperone, meperidina, cetamina e xilazina, foi similar a empregada em pacas [8], para a realização de hemi-ovariossalpingohisterectomia, na qual os autores empregaram azaperone, atropina, cetamina, xilazina e alotano e obtiveram boa analgesia cirúrgica.

\begin{tabular}{|c|c|c|c|c|c|c|c|c|c|}
\hline & Cutia 1 & Cutia 2 & Cutia 3 & Cutia 4 & Cutia 5 & Cutia 6 & Cutia 7 & $\begin{array}{l}\text { Média } \\
\text { Total }\end{array}$ & $\begin{array}{l}\text { Desvio } \\
\text { Padrão }\end{array}$ \\
\hline $\begin{array}{l}\text { Temperatura Retal } \\
\text { normal }\left({ }^{\circ} \mathrm{C}\right)\end{array}$ & 36,0 & 38,0 & 38,9 & 39,2 & 38,6 & 38,0 & 38,9 & 38,2 & 1,08 \\
\hline $\begin{array}{l}\text { Diminuição da } \\
\text { temperatura retal }\left({ }^{\circ} \mathrm{C}\right)\end{array}$ & 1,9 & 2,3 & 1,2 & 0 & 0 & 2,2 & 1,2 & 1,76 & 0,53 \\
\hline Latência Inicial (min) & 7,0 & 3,0 & 4,0 & 4,0 & 3,0 & 2,0 & 4,0 & 3,86 & 1,87 \\
\hline Latência Final (min) & 7,0 & 6,0 & 6,0 & 6,0 & 6,0 & 13,0 & 4,0 & 6,86 & 2,85 \\
\hline Tempo de analgesia (min) & 107 & 84 & 84 & 55 & 68 & 84 & 84 & 80,86 & 16,1 \\
\hline
\end{tabular}


As doses empregadas foram adaptadas do suíno para o azaperone [6], dos roedores selvagens no caso da cetamina e xilazina [9], e do cão, em relação à meperidina e lidocaína [6], diferentemente de autores que adotaram apenas o cão como modelo de comparação para as doses empregadas nas cutias [1].

Foi obtida boa analgesia com a anestesia epidural pela lidocaína, assim como o descrito para roedores [6]. O período hábil, de $80,86 \pm 16,1$ minutos, encontra-se dentro da faixa de variação de uma a duas horas descrita para os cães [4], mas é superior ao período de 30 minutos descrito em ratos [6].

Houve diminuição da temperatura corporal nas cutias anestesiadas, similarmente ao já descrito nessa espécie [1,7], e em capivaras [3].

\section{CONCLUSÕES}

A técnica de anestesia balanceada empregada proporcionou analgesia adequada, durante um período que seria suficiente para a realização de procedimentos clínicos, diagnósticos ou cirúrgicos, com a utilização de doses reduzidas de xilazina e cetamina.

\section{NOTAS INFORMATIVAS}

${ }^{1}$ Stresnil ${ }^{\circledR}$ - Janssen Pharmaceutica, São Paulo, SP/Brasil.

${ }^{2}$ Dolossal ${ }^{\circledR}$ - Cristália Produtos Quimicos e Farmaceuticos Ltda., Itapira, SP/Brasil.

${ }^{3}$ Coopazine ${ }^{\circledR}$ - Schering-Plough Coopers Brasil Ltda., São Paulo, SP/Brasil.

${ }^{4}$ Vetaset $^{\circledR}$ - Fort Dodge Saúde Animal Ltda., Campinas, SP/ Brasil.

${ }^{5}$ Lidovet $^{\circledR}$ - Bravet Ltda., Rio de Janeiro, RJ/Brasil.

\section{REFERÊNCIAS}

1 Araujo R.W., Feitosa JR. F.S. \& Fernandes R.M. 2005. Efeitos cardiorespiratórios da associação tiletamina-zolazepam comparada com a associação tiletamina-zolazepam, atropina, xilazina através da extrapolação alométrica em cutias (Dasyprocta sp). In: Resumos do IX Congresso e XIV Encontro da Associação Brasileira de Veterinários de Animais Selvagem - ABRAVAS (São José do Rio Preto, Brasil) p.60.

2 Chaves J.W.N. \& Santos J.H.R. 2005. Disponível em: <http://www.esam.br/zoobotanico/animais/cutia.htm>. Acessado em 21 jun 2005.

3 Cruz M.L., Luna, S.P.L., Moura, C.A., Castro, G.B., Teixeira Neto F.J. \& Nishida S.M. 1998. Técnicas Anestésicas Injetáveis em Capivaras (Hydrochoerus hydrochaeris). Ciência Rural. 28: 411-415.

4 Intelizano T.R, Santos P.R, Futema F, Otsuki D.A. \& Almeida T.I. 2002. Técnicas de anestesia local - anestesia epidural. In: Fantoni D.T. \& Cortopassi S.R. (Eds). Anestesia em cães e gatos. São Paulo: Roca, pp.190-208.

5 Massone F. 2003. Anestesiologia Veterinária: Farmacologia e Técnicas. 4.ed. Rio de Janeiro: Guanabara Koogan S.A, 252p.

6 Massone F. 2003. Atlas de Anestesiologia Veterinária. São Paulo: ROCA, 188p.

7 Moura S.G., Fernandes R.M., Silva Júnior J.R., Barradas A.L.B., Araújo W.R., Carvalho M.A.M. \& Lopes J.B. 2002. Cloridrato de tiletamina associado com cloridrato de zolazepam na tranquilização e anestesia de cutias (Dasyprocta sp). In: VI Congresso e XI Encontro da Associação Brasileira de Veterinários de Animais Selvagens (Guarapari, Brasil). p.70.

8 Oliveira F.S., Toniollo G.H., Machado M.R.F. \& Paura D. 2003. Hemi-ovariossalpingohisterectomia em pacas prenhes e posterior ocorrência de prenhez (Agouti paca, Linnaeus, 1766). Ciência Rural. 33: 547-551.

9 Sainsbury A.W. 2003. Rodentia In: Fowler M.E \& Miller R.E. (Eds). Zoo and Wild Animal Medicine. 5th edn. St Louis: Saunders, pp.420-442. 